\title{
CHARGE-BASED MOS CORRELATED DOUBLE SAMPLING COMPARATOR AND FOLDING CIRCUIT
}

\author{
Roman Genov and Gert Cauwenberghs \\ Department of Electrical and Computer Engineering \\ The Johns Hopkins University, Baltimore, MD 21218-2686 \\ E-mail: \{roman, gert\}abach.ece.jhu .edu
}

\begin{abstract}
A novel charge-based comparator and folding circuit are presented. Correlated double sampling comparison is performed using a log-domain integrator, implemented by a subthreshold $n$ MOS transistor with the source coupled to a capacitor. The circuit produces a current that is a logistic function of the change in voltage on the gate, with an input-referred offset voltage that is a logarithmic function of time. Folding operation for analogto-digital conversion is obtained by differentially combining currents from a bank of these comparators. A prototype 128-channel parallel 4-bit gray-code analog-to-digital converter has been implemented in a $0.5 \mu \mathrm{m}$ CMOS process, delivering $128 \mathrm{MS} / \mathrm{sec}$ at $76 \mathrm{~mW}$ power dissipation.
\end{abstract}

\section{INTRODUCTION}

High-performance data conversion can be achieved either by expending power and area to achieve high precision in a single ana$\log$ architecture, or by distributing the architecture over multiple low-resolution quantization tasks each implemented with relatively imprecise analog circuits, and combined in the digital domain. The latter approach has proven superior in attaining very high precision, by distributing the quantization process over time using delta-sigma modulation [1]. Both high speed and high resolution can be achieved by distributing the quantization process in space. To this end, it is necessary to implement very space efficient, low-resolution quantizers.

We present a charge-based circuit that implements an offsetcompensated comparator with one capacitor and four $n$ MOS transistors. Besides applications in data conversion, the design targets applications in hybrid analog-digital computing using large-scale analog arrays [2], where parallelism, redundancy in information representation, and statistical data coding [3] can be used to compensate for imperfections in analog sensing and computation.

\section{CHARGE-DOMAIN CORRELATED DOUBLE SAMPLING COMPARATOR}

\subsection{Capacitor- $n$ MOS Integrator}

To obtain high density and high speed in a comparator and folding circuit, the challenge is to design a single stage producing a current-mode or charge-mode signal that is a saturating high-gain and offset-compensated function of a difference in input voltage.

\footnotetext{
This work was supported by ONR N00014-99-1-0612
}

We show that in the charge domain this can be achieved using a circuit incorporating a capacitor and an exponential element, such as a diode [4] or a MOS transistor operating in subthreshold regime [5], where the differential voltage is presented as an initial condition at the input. Offset compensation is achieved in the charge domain, as for the CMOS charge-transfer comparator described in [6].

In the circuit of Figure $1(a)$ the $n$ MOS transistor is source coupled to a capacitor. In the subthreshold and saturation region, the drain current is exponential in gate and source voltage, and the large-signal dynamics of the integrator are described by:

$$
C \frac{d V_{s}}{d t}=I_{s}=\frac{W}{L} I_{o} e^{\left(\kappa V_{g}-V_{s}\right) / V_{t}}
$$

where $V_{t}$ is the thermal voltage. Integrating the differential equation (1) yields:

$$
C V_{t} e^{\frac{V_{s}}{V_{t}}}=\frac{W}{L} I_{o} e^{\kappa V_{g} / V_{t}} t+c_{1}
$$

where $c_{1}$ is an integration constant. Direct substitution yields:

$$
I_{s}(t)=\frac{C V_{t}}{t+\frac{c_{1}}{\frac{W}{L} I_{o} e^{\kappa V g / V_{t}}}} .
$$

At time $t=0$, the input voltage is switched from $V_{g}\left(0^{-}\right)$to $V_{g}\left(0^{+}\right)$while the capacitor instantly retains the source voltage $V_{s}(0)$. The source current therefore switches from $I_{s}\left(0^{-}\right)$to $I_{s}\left(0^{+}\right)$over the transition at the gate:

$$
I_{s}\left(0^{+}\right)=I_{s}\left(0^{-}\right) e^{\kappa \Delta V_{g} / V_{t}},
$$

where $\triangle V_{g}=V_{g}\left(0^{+}\right)-V_{g}\left(0^{-}\right)$. The output current (3) can thus be expressed in terms of initial conditions:

$$
I_{s}(t)=\frac{I_{s}(0+)}{\frac{I_{s}(0+)}{C V_{t}} t+1}=\frac{I_{s}(0-)}{\frac{I_{s}(0-)}{C V_{t}} t+e^{-\kappa \Delta V_{g} / V_{t}}} .
$$

Interestingly, for $t \gg C V_{t} / I_{s}\left(0^{+}\right), I_{s}(t)$ becomes independent of initial conditions:

$$
I_{s}(t) \approx \frac{C V_{t}}{t}
$$

\subsection{Comparator}

Saturation of the output current of the circuit in Figure $1(a)$ as a function of a change in the input voltage $V_{g}$ is utilized in the design of the charge-based comparator as shown in Figure 1(b). The 


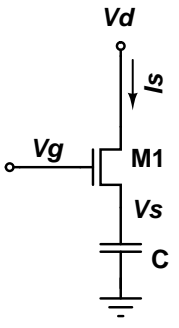

(a)

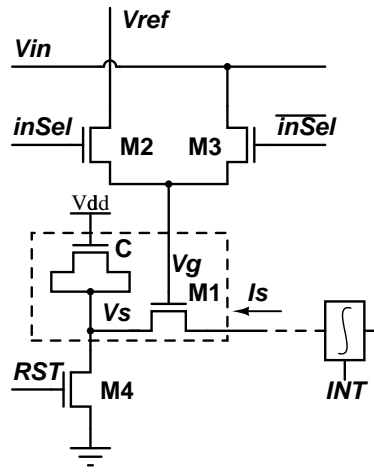

(b)
Figure 1: (a) Capacitor-nMOS integrator and (b) charge-based comparator.

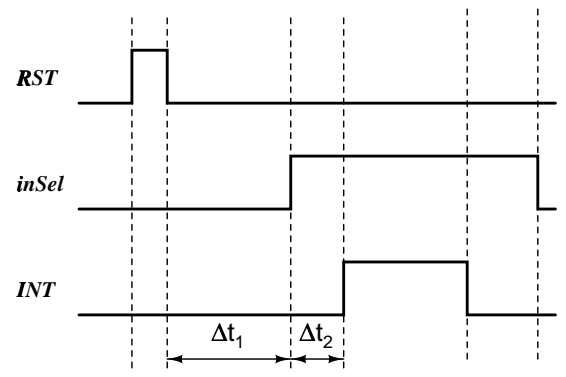

Figure 2: Control signal timing diagram for the comparator circuit in Figure 1 (b).

$n$ MOS capacitor is initially charged by pulsing $R S T$ as shown in Figure 2. Over a time interval $\Delta t_{1}$, the capacitor discharges to raise $V_{s}$ until $M 1$ reaches well into the subthreshold region. The end of the interval defines the initial condition for the source current $I_{s}\left(0^{-}\right)$. The differential input voltage is presented as a transient on the gate, $\triangle V_{g}=V_{\text {ref }}-V_{\text {in }}$, implemented using an analog multiplexer M2-M3 and controlled by in Sel/inSel timing signals in Figure 2. In subthreshold ${ }^{1}$, this gate voltage transition produces a change in source current according to (5). By combining equations (4) and (5), the input-output characteristic of the comparator can be expressed as:

$$
I_{s}(t)=I_{s a t} \sigma\left(A\left(\triangle V_{g}-V_{\text {off }}(t)\right)\right),
$$

where

$$
\sigma(x)=\frac{1}{1+e^{-x}}
$$

is a logistic function, the amplitude saturates to $I_{s a t}=C V_{t} / t$, the voltage is scaled by $A=\kappa / V_{t}$, and the offset voltage

$$
V_{\text {off }}(t)=\frac{V_{t}}{\kappa} \log \frac{C V_{t}}{I_{s}\left(0^{-}\right) t}
$$

is a logarithmic function of time. Note that by virtue of correlated double sampling in the differential transient $\triangle V_{g}$ by switching

\footnotetext{
${ }^{1}$ For large values of $\triangle V_{g}$, the $n$ MOS may initially enter the strong inversion region. This affects the timing but not the operation of the circuit, since once the capacitor has raised $V_{s}$ to reach subthreshold, the asymptotic relationship (6) holds again.
}

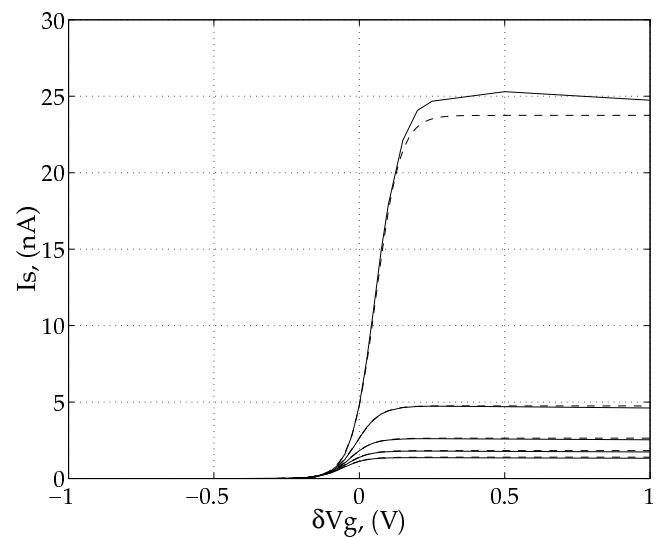

Figure 3: The input-output characteristics of the charge-based comparator at different fixed time intervals $\triangle t_{2}$ after switching the inputs, calculated using equation (7) (dashed line) and simulated using SpectreS for a $0.5 \mu \mathrm{m}$ CMOS process (solid line). (Top to bottom: $\triangle t_{2}=50,250,450,650,850 \mathrm{~ns}$.)

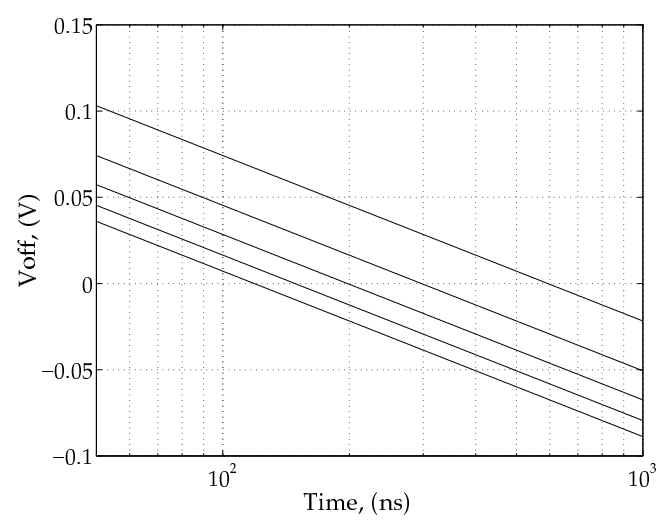

Figure 4: The offset voltage of the comparator as a function of time after switching the inputs ( $\triangle t_{2}$ in Figure 2). Theoretical results obtained using equation (9) for different values of $I_{s}\left(0^{-}\right)$. (Top to bottom: $I_{s}\left(0^{-}\right)=2,4,6,8,10 n A$.)

M2-M3, the offset $V_{\text {off }}(t)$ is independent of M1 threshold variations and, to first order, $1 / f$ noise.

Figure 3 illustrates the input-output characteristics of the comparator for different time interval $\Delta t_{2}$ after switching the inputs, calculated using equation (7) and simulated using SpectreS for a $0.5 \mu \mathrm{m}$ CMOS process. The offset of the comparator as a function of time from (9) is plotted in Figure 4. It scales logarithmically in time. It also depends on $I_{s}\left(0^{-}\right)$which is controlled by the time interval, $\triangle t_{1}$, between the moment $V_{\text {in }}$ is applied (after the reset) and the time $V_{\text {ref }}$ is presented to the gate of $M 1$. Figure 5 illustrates theoretical and simulated source current transients for different values of $\triangle V_{g}$.

\section{CHARGE-BASED FOLDING CIRCUIT AND GRAY-CODE ADC}

Several solutions for gray-code and charge-based A/D conversion exist. Conventional folded differential logic [7] (FDL) can elimi- 


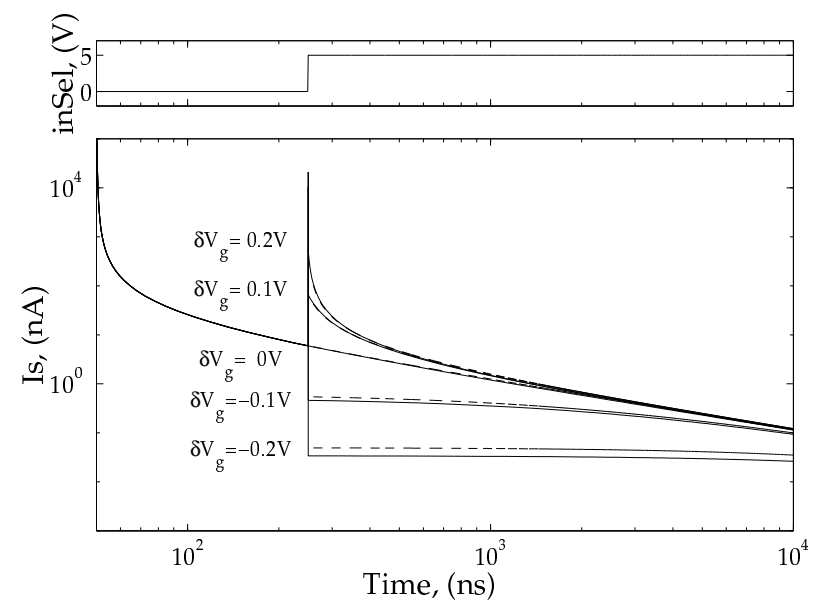

Figure 5: Comparator output current transients for different values of $\triangle V_{g}$. The solid line shows the SpectreS simulation results for a $0.5 \mu \mathrm{m}$ CMOS process; the dashed line was obtained using equation (7). The initial current $I_{s}\left(0^{-}\right)=6 n$ A. Effective $n M O S$ capacitor value is $45 f F$.

nate code errors due to its wired gray-code encoding scheme. An improvement of FDL, folding cascoded differential logic (FCDL), was introduced in [8]. It allows for higher number of comparators in an encode block.

\subsection{Gray-Code Flash ADC Architecture}

Figure 6(a) shows the architecture of a 3-bit gray-code differential logic ADC. Comparators produce the output current

$$
I_{s}^{n}=I_{b}\left(\left(f\left(V_{\text {ref }}^{n}-V_{\text {in }}\right)+1\right) / 2\right),
$$

where $I_{b}$ is a bias current, $V_{\text {ref }}{ }^{n}$ is a respective reference voltage level between $V_{\text {ref }}{ }^{\min }$ and $V_{\text {ref }}{ }^{\max }, n=1, \ldots, 7$, and $f($.$) is a de-$ cision function such as $\operatorname{sign}($.$) or a logistic function. Each output$ bit, $D_{i}, i=0, \ldots, 2$, is obtained by connecting comparator outputs differentially in a folding circuit, and then comparing the accumulated differential currents. The input-output characteristics of the ADC are illustrated in Figure 6(b).

\subsection{Folding Circuit}

An LSB folding circuit for a 5-bit flash ADC is shown in Figure 7. The output currents can be expressed as:

$$
\begin{gathered}
I_{+}=I_{\text {sat }} \sum_{n=0}^{(N-1) / 4} \sigma\left(A\left(V_{\text {in }}-V_{\text {ref }}^{4 n+1}-V_{\text {off }}(t)\right)\right), \\
I_{-}=I_{\text {sat }} \sum_{n=0}^{(N-1) / 4} \sigma\left(A\left(V_{\mathrm{in}}-V_{\text {ref }}^{4 n+3}-V_{\text {off }}(t)\right)\right)+I_{b} / 2,
\end{gathered}
$$

where

$$
I_{b}=I_{s a t} \sigma\left(A\left(V_{\mathrm{ref}}^{\max }-V_{\mathrm{ref}}^{\mathrm{min}}-V_{\mathrm{off}}(t)\right)\right) .
$$

Theoretical and simulated output currents as a function of the input voltage for a folding circuit of a flash ADC are demonstrated in Figure 8.

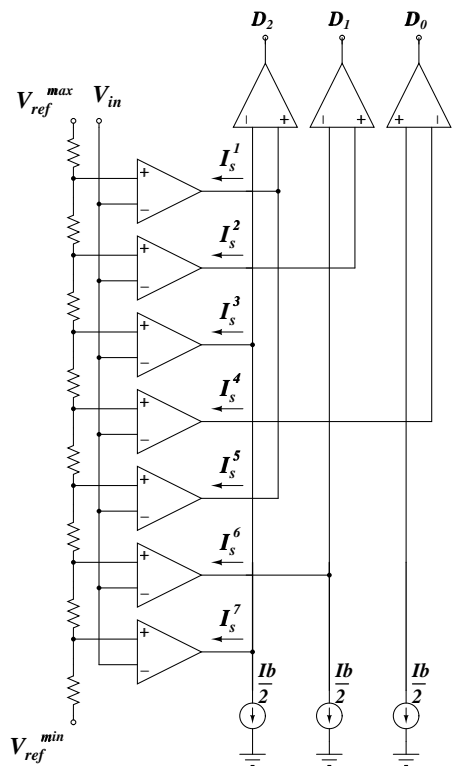

(a)

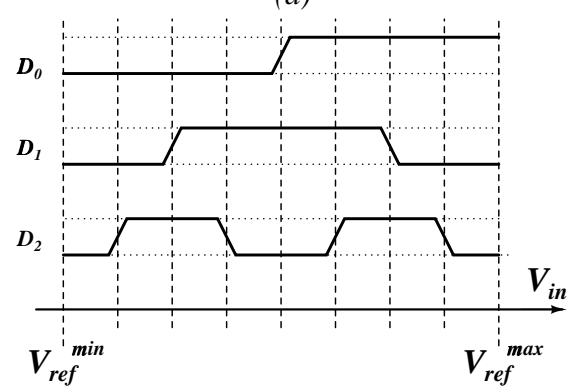

(b)

Figure 6: A 3-bit gray-code flash A/D converter: (a) architecture; (b) characteristic.

\subsection{Integrating Sense Amplifier}

Bit decisions are made on integrated, differentially folded comparator currents using a regenerative sense amplifier. Integration starts at the end of the $\triangle t_{2}$ interval, timed by the $I N T$ clock signal in Figure 2. The time-dependent comparator voltage offset (9) is inconsequential to the integration as it is in common to all comparator cells.

\section{EXPERIMENTAL RESULTS}

A prototype 128-channel 4-bit charge-based gray code ADC was fabricated in a $0.5 \mu \mathrm{m}$ CMOS process. The die micrograph is shown in Figure 9. The chip contains, besides the parallel bank of flash ADCs, a massively parallel mixed-signal computational array [9]. The ADC bank measures $0.75 \mathrm{~mm} \times 2 \mathrm{~mm}$, and dissipates $76 \mathrm{~mW}$ of power at $128 \mathrm{MS} / \mathrm{sec}$ sampling rate. Output waveforms for a ramp input signal are presented in Figure 10.

\section{CONCLUSIONS}

A new charge-based comparator and a folding circuit have been reported. Each comparator performs correlated double sampling 


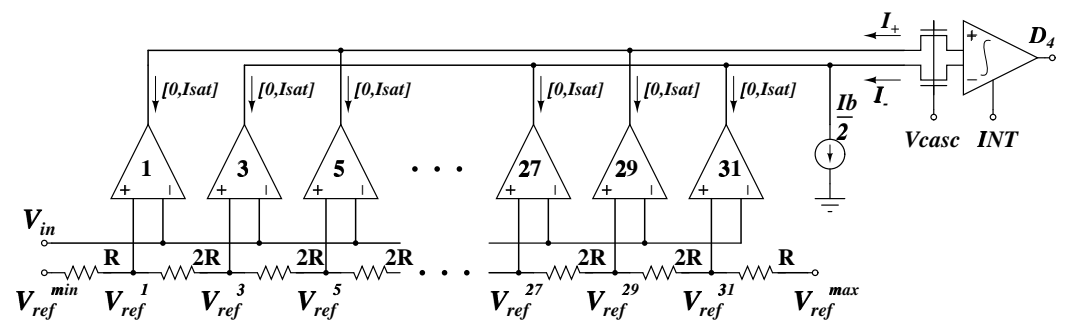

Figure 7: Folding circuit for a charge-based gray code flash ADC.

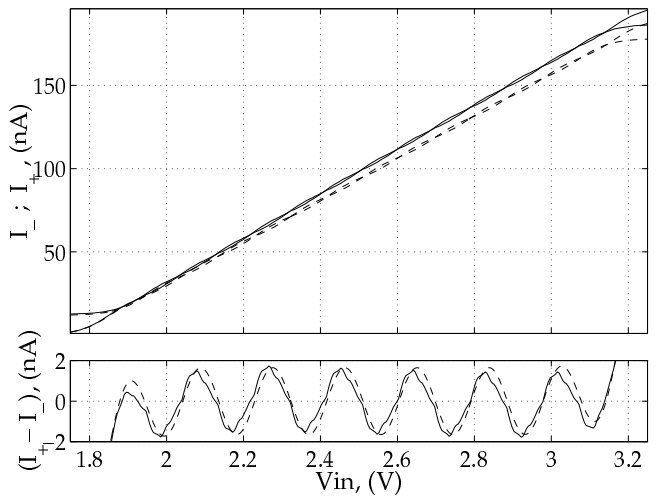

Figure 8: Output currents of the folding circuit of Figure 7. The solid line represents the SpectreS simulation results for a $0.5 \mu \mathrm{m}$ CMOS process; the dashed line corresponds to equations (11) and (12). The time interval $\triangle t_{2}=50 \mathrm{~ns}$.

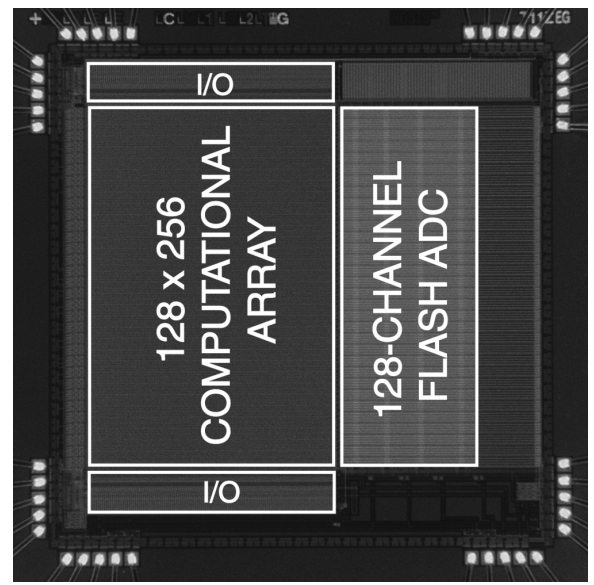

Figure 9: Micrograph of a mixed-signal processor containing a computational array and a 128-channel parallel 4-bit gray-code flash analog-to-digital converter. Die size is $3 \mathrm{~mm} \times 3 \mathrm{~mm}$ in $0.5 \mu \mathrm{m}$ CMOS technology.

of the inputs to avoid mismatch errors. The circuit operates in weak inversion and yields both high speed and low power. The design is suited for parallel data conversion on mixed-signal computational arrays, active pixel imagers, and other distributed charge or voltage mode circuits. A prototype 128-channel parallel 4-bit gray-code analog-to-digital converter has been implemented in a

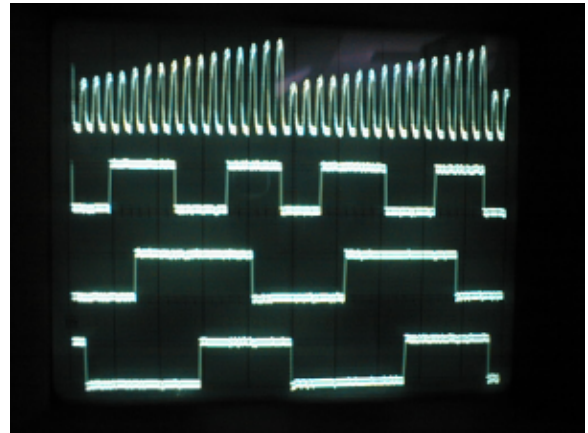

Figure 10: Recorded gray-code flash ADC output waveforms as a function of input voltage.

$0.5 \mu \mathrm{m}$ CMOS process, delivering $128 \mathrm{MS} / \mathrm{sec}$ at $76 \mathrm{~mW}$ power dissipation. It is trivial to extend the circuit with bipolar junction transistors for use in RF ADCs.

\section{REFERENCES}

[1] J.C. Candy and G.C. Temes, "Oversampled Methods for A/D and D/A Conversion," in Oversampled Delta-Sigma Data Converters, IEEE Press, pp 1-29, 1992.

[2] R. Genov, G. Cauwenberghs "Charge-Mode Parallel Architecture for Matrix-Vector Multiplication," to appear in IEEE T. Circuits and Systems II, vol. 48 (10), 2001.

[3] R. Genov, G. Cauwenberghs, "Stochastic Mixed-Signal VLSI Architecture for High-Dimensional Kernel Machines," to appear in Advances in Neural Information Processing Systems, Cambridge, MA: MIT Press, vol. 14, 2002.

[4] R. Sarpeshkar, J. Kramer, et. al., "Analog VLSI Architectures for Motion Processing: From Fundamental Limits to System Applications," Proc. of The IEEE, vol. 84 (7), pp. 969-987, 1996.

[5] K. Boahen, "Retinomorphic Vision Systems," Proc. of 5th Int. Conf. MicroNeuro'96, 1996

[6] K. Kotani, T. Shibata, et. al., "CMOS Charge-Transfer Preamplifier for Offset-Fluctuation Cancellation in Low-Power Converters," IEEE J. Solid-State Circuits, vol. 33 (5), pp. 762-769, 1998.

[7] H. Kimura, A. Matsuzawa, T. Nakamura "A $10 \mathrm{~b} 300 \mathrm{MHz}$ Interpolated-Parallel A/D Converter," IEEE T. Circuits and Systems II, vol. 44 (2), pp. 65-85, 1997.

[8] K. Ono, T. Matsuura, et. al., "Error Suppressing Encode Logic of FDCL in 6-bit Flash A/D Converter," IEEE J. Solid-State Circuits, vol. 32 (9), pp. 1460-1464, 1997.

[9] R. Genov, G. Cauwenberghs "Massively Parallel Inner-Product Array Processor," Proc. of Int. Joint Conference on Neural Networks, vol. 1, pp. 183-188, 2001. 